\title{
Zinc Deprivation Mediates Alcohol-Induced Hepatocyte IL-8 Analog Expression in Rodents via an Epigenetic Mechanism
}

\author{
Yantao Zhao, ${ }^{{ }^{\dagger}}$ Wei Zhong, ${ }^{{ }^{\dagger}}$ Xiuhua Sun, ${ }^{\text {† }}$ \\ Zhenyuan Song, ${ }^{\S}$ Dahn L. Clemens, ${ }^{\text {I }}$ \\ Y. James Kang, ${ }^{+\|}$Craig J. McClain, ${ }^{* \dagger \mid \star * *}$ \\ and Zhanxiang Zhou ${ }^{\dagger \dagger}$ \\ From the Departments Medicine* and Pharmacology \& \\ Toxicology," the University of Louisville Alcohol Research Center, ${ }^{\dagger}$ \\ and the Louisville Veterans Affairs Medical Center,"* Louisville, \\ Kentucky; the Department of Kinesiology, ${ }^{\S}$ University of Illinois at \\ Chicago, Chicago, Illinois; the Department of Internal Medicine, " \\ University of Nebraska Medical Center, Omaha, Nebraska; and \\ the Department of Nutrition, ${ }^{+1}$ University of North Carolina at \\ Greensboro, Greensboro, North Carolina
}

Neutrophil infiltration caused by IL-8 production is a central mechanism in alcohol-induced hepatitis. This study was performed to examine if an epigenetic mechanism is involved in alcohol-induced IL-8 production. Mice were pair-fed an alcohol-containing liquid diet for 4 weeks. Alcohol exposure induced hepatitis as indicated by increased expression of keratinocyte-derived cytokine (mouse IL-8) and neutrophil infiltration. Alcohol exposure induced histone 3 hyperacetylation owing to inhibition of histone deacetylase (HDAC) in association with NF- $\kappa$ B activation. Cell culture studies showed that alcohol exposure induced IL-8 and cytokineinduced neutrophil chemoattractant-1 (CINC-1, rat IL-8) production in human VL-17A cells and rat H4IIEC3 cells, respectively, dependent on acetaldehyde production, oxidative stress, and zinc release. Zinc deprivation alone induced CINC-1 production and acted synergistically with lipopolysaccharide or tumor necrosis factor- $\alpha$ on CINC-1 production. Zinc deprivation induced histone 3 hyperacetylation at lysine 9 through suppression of HDAC activity. Zinc deprivation caused nuclear translocation of NF- $\kappa B$, and reduced HDAC binding to NF- $\kappa B$. Chromatin immunoprecipitation (ChIP) showed that zinc deprivation caused histone 3 hyperacetylation as well as increased NF- $\mathrm{B}$ binding to the CINC-1 promoter. In conclusion, inactivation of HDAC caused by zinc deprivation is a novel mechanism underlying IL-8 up-regulation in alcoholic hepatitis. (Am J Pathol 2011, 179:693-702; DOI: 10.1016/j.ajpath.2011.04.006)

Neutrophil infiltration is well documented in patients with alcoholic hepatitis and experimental animals with alcohol-induced liver injury. ${ }^{1-3}$ Neutrophils can cause liver tissue damage through releasing reactive oxygen species (ROS) and proteases. ${ }^{3} \mathrm{CXC}$ chemokines play a central role in chemoattraction of neutrophils, and $\mathrm{IL}-8$ (CXCL-8) has been suggested as a major CXC chemokine in mediating alcohol-induced neutrophil infiltration in the liver. ${ }^{4,5}$ Patients with alcoholic hepatitis showed an increased IL-8 level in both serum and the liver. ${ }^{6-8}$ The serum IL-8 levels in alcoholic liver disease were correlated closely with severity of liver injury. ${ }^{7,8}$ In addition, hepatic local IL-8 levels correlated with the degree of neutrophil infiltration. ${ }^{7,8}$ Mouse keratinocyte-derived cytokine (KC) and rat cytokine-induced neutrophil chemoattractant-1 (CINC-1) are analogs of human IL-8. Chronic alcohol feeding has been shown to up-regulate $\mathrm{KC} / \mathrm{CINC}$ in association with neutrophil infiltration in the liver. ${ }^{1,9,10} \mathrm{~A}$ variety of cell populations in the liver are known to produce IL-8 in response to alcohol consumption. However, the mechanisms of IL-8 production in hepatocytes are poorly understood, although it has been shown repeatedly by both in vivo and in vitro studies..$^{8,11-16}$

$\mathrm{NF}-\kappa \mathrm{B}$ is a key transcription factor in up-regulation of cytokine/chemokine genes in alcoholic liver disease. ${ }^{4,5}$ Increasing evidence suggests that NF- $\kappa \mathrm{B}-$ mediated

Supported in part by National Institutes of Health grants R01AA016013 (Z.Z.), and R01R37AA010762, P01 AA017103, R01AA 015970, R01DK071765, R01AA018869, R01AA018016, P30AA019360, RC2AA019385, and P30ES014443-01A1 (C.J.M.); and the Veterans Administration (C.J.M.). Y.J.K. and C.J.M. are Distinguished University Scholars of the University of Louisville.

Accepted for publication April 25, 2011.

Y.Z. and W.Z. contributed equally to this work.

Address reprint requests to Zhanxiang Zhou, Ph.D., Department of Nutrition, University of North Carolina at Greensboro, 500 Laureate Way, Suite 4226, Kannapolis, NC 28081. E-mail: z_zhou@uncg.edu. 
gene transcription is critically regulated by acetylation of core histones. ${ }^{17-19}$ Histone acetylation is controlled by histone acetyltransferases (HATs) and histone deacetylases (HDACs). ${ }^{20}$ HATs promote histone acetylation and open up chromatin, thereby facilitating gene transcription. In contrast, HDACs remove acetyl groups from histones and repack chromatin, thereby terminating gene transcription. Recent studies showed that HDACs have a significant impact on NF- $\kappa \mathrm{B}-$ mediated IL-8 expression in inflammatory lung disease. ${ }^{21-23}$ Pharmaceutical inhibition of HDACs promoted DNA binding activity of NF- $\kappa \mathrm{B}$ and IL-8 production in alveolar epithelial cells. ${ }^{24-26} \mathrm{Re}-$ cent studies also suggested an emerging role of epigenetics in alcohol-induced liver damage. ${ }^{27}$ Acute alcohol intoxication has been shown to induce histone hyperacetylation at lysine 9 in the liver via increasing HAT activity. ${ }^{28-31}$ However, the role of epigenetic regulation of $\mathrm{NF}-\kappa \mathrm{B}-$ mediated IL-8 expression in the liver has not been defined.

The deacetylase activity of all classic HDACs (class I, $\mathrm{II}$, and IV) is zinc dependent. ${ }^{20}$ Alcohol consumption has been shown to cause zinc deficiency in the liver of alcoholic patients and experimental animals. ${ }^{32-34}$ Our previous study showed that chronic alcohol exposure to mice induced hepatic keratinocyte chemoattractant (KC, mouse (L-8), which was attenuated by dietary zinc supplementation. ${ }^{9}$ In the present study, we show that inactivation of HDACs owing to zinc deprivation is an important mechanism of alcohol-induced IL-8 expression in hepatocytes.

\section{Materials and Methods}

\section{Animals and Alcohol Feeding Procedure}

Male C57BL/6J mice were obtained from Harlan (Indianapolis, IN). All of the mice were treated according to the experimental procedures approved by the Institutional Animal Care and Use Committee. For chronic alcohol exposure, at 4 months of age the mice were pair-fed a modified Lieber-DeCarli alcohol or isocaloric maltose dextrin control liquid diet for 4 weeks with a stepwise feeding procedure as described previously. ${ }^{35}$ The ethanol content $(\%, w / v)$ in the diet was $4.8(34 \%$ of total calories) at initiation, and gradually increased up to 5.4 (38\% of total calories). The amount of food given to the pair-fed mice was that of alcohol-fed mice measured the previous day. At the end of the feeding experiment, mice were anesthetized, and blood and liver tissues were collected.

\section{Hepatoma Cell Culture}

VL-17A cells, ${ }^{36}$ a recombinant HepG2 cell line expressing alcohol metabolic enzymes, and H4IIEC3 rat hepatoma cells (American Type Culture Collection, Rockville, MD) were grown in Dulbecco's modified Eagle medium (Invitrogen, Carlsbad, CA) supplemented with 10\% fetal bovine serum and penicillin (100 U/mL)/streptomycin sulfate $(100 \mu \mathrm{g} / \mathrm{mL})$ (Invitrogen). Alcohol-induced IL-8 production was determined in both VL-17A cells and
H4IIEC3 cells. Cells were treated with alcohol at 50 $\mathrm{mmol} / \mathrm{L}$ for 24 hours with or without adding $\mathrm{N}$-acetylcysteine (NAC) at $2 \mathrm{mmol} / \mathrm{L}$ or $50 \mu \mathrm{mol} / \mathrm{L}$ zinc sulfate 30 minutes before alcohol treatment. To define the role of zinc deprivation in IL-8 production, H4IIEC3 cells were treated with a zinc chelator, $\mathrm{N}, \mathrm{N}, \mathrm{N}^{\prime}, \mathrm{N}^{\prime}$-tetrakis (2-pyridylmethyl) ethylenediamine (TPEN) at $1 \mu \mathrm{mol} / \mathrm{L}$ for 3 days. To determine the interaction between zinc deprivation and LPS or tumor necrosis factor (TNF)- $\alpha$ in IL-8 expression, H4IIEC3 cells were treated with TPEN for 2 days, followed by treatment with LPS at $100 \mathrm{ng} / \mathrm{mL}$ or TNF- $\alpha$ at $0.1 \mathrm{ng} / \mathrm{mL}$ for 24 hours. To confirm the specificity of zinc chelating action by TPEN, zinc sulfate was added at 50 $\mu \mathrm{mol} / \mathrm{L}$ zinc ion with TPEN treatment.

\section{IHC}

For immunohistochemistry (IHC), liver tissues were fixed with $10 \%$ formalin and cut into $5-\mu \mathrm{m}$ sections. For detection of neutrophils, the sections were incubated with monoclonal rat anti-mouse Ly-6G (BD Pharmingen, San Diego, CA) at $4^{\circ} \mathrm{C}$ overnight, followed by incubation with a horseradish-peroxidase-conjugated anti-rat IgG for 30 minutes. For detection of histone 3 acetylation at lysine 9 , sections were incubated with rabbit acetyl-histone H3 (Lys9) antibody (Cell Signaling, Danvers, MA) at $4^{\circ} \mathrm{C}$ overnight, followed by incubation with a horseradish-peroxidase-conjugated anti-rabbit IgG for 30 minutes. Diaminobenzidine was used as horseradish-peroxidase substrate for visualization. The negative controls were conducted by omitting the primary antibody.

\section{ELISA}

Liver proteins were extracted with radioimmunoprecipitation assay buffer, and $\mathrm{KC}$ levels were measured using a $\mathrm{KC}$ enzyme-linked immunosorbent assay kit (ELISA) (R\&D systems, Minneapolis, MN). IL-8 or CINC-1 concentrations in cell culture media were determined by ELISA with an IL-8 or CINC-1 kit (R\&D Systems).

\section{HAT Activity}

HAT activity was measured using a fluorimetric assay kit (Active Motif, Carlsbad, CA) following the manufacturer's instructions. In brief, the HAT protein was incubated with acetyl-CoA and either a histone $\mathrm{H} 3$ or histone $\mathrm{H} 4$ substrate peptide. During this reaction, the HAT enzyme catalyzes the enzymatic transfer of acetyl groups from the acetyl-CoA to the histone peptide, thereby generating the acetylated peptide and CoA. The reaction is stopped by adding stop solution, and after adding the complete developing solution the developer reacts with the free sulfhydryl groups on the CoA to give a fluorescent reading of acetyltransferase activity.

\section{HDAC Activity}

HDAC activity was measured with a fluorimetric assay kit (Biomol, Plymouth Meeting, PA) according to the manu- 
facturer's instructions. The procedure involves the use of HDAC fluorimetric substrate (Fluor de Lys substrate), which comprises an acetylated lysine side chain and is incubated with a sample containing nuclear extract. Deacetylation sensitizes the substrate, which then generates a fluorophore. The fluorophore is excited with 360 $\mathrm{nm}$ light and emitted with $460 \mathrm{~nm}$ light.

\section{Measurement of Acetaldehyde}

H4IIEC3 and VL-17A cells were seeded into flasks, respectively. After 24-hours of incubation, the medium was replaced with an equal volume of DMEM containing 25 $\mathrm{mM}$ HEPES and $50 \mathrm{mmol} / \mathrm{L}$ ethanol. The flasks were tightly sealed and incubated at $37^{\circ} \mathrm{C}$ for another 24 hours. Acetaldehyde concentrations in the media were measured with a kit (Rbiopharm, Germany) following manufacturer's instructions.

\section{Measurement of ROS}

ROS accumulation in VL-17A/H4IIEC3 cells was examined by measuring the dihydroethidium fluorescence intensity. Nonfluorescent dihydroethidium is oxidized by ROS to yield the red fluorescent product, ethidium, which binds to nucleic acids, staining the nucleus a bright fluorescent red. VL-17A/H4IIEC3 cells were incubated with $5 \mu \mathrm{mol} / \mathrm{L}$ dihydroethidium (Molecular Probes, Eugene, OR) for 30 minutes at $37^{\circ} \mathrm{C}$ in the dark. The ROS-catalyzed ethidium red fluorescence was examined with an excitation wavelength of $544 \mathrm{~nm}$ and an emission wavelength of $590 \mathrm{~nm}$.

\section{Measurement of Cellular Free Zinc}

Cellular free zinc was detected by a green fluorescent zinc ion indicator (FluoZin-3; Molecular Probes). Cells were incubated with $5 \mu \mathrm{mol} / \mathrm{L}$ FluoZin-3 at $37^{\circ} \mathrm{C}$ for 30 minutes in the dark. The generated fluorescence signal was read by a fluorescence spectrophotometer (Synergy HT Multi-Mode Microplate Reader; BioTek, Winooski, VT) with an excitation wavelength of $494 \mathrm{~nm}$ and an emission wavelength of $516 \mathrm{~nm}$.

\section{Histone Extraction}

For nuclear histone lysate preparation, after treatment cells were washed twice with ice-cold PBS containing 5 $\mathrm{mmol} / \mathrm{L}$ sodium butyrate, scraped and lysed in hypotonic lysis buffer (20 mmol/L HEPES, $1 \mathrm{mmol} / \mathrm{L}$ EDTA, 10 $\mathrm{mmol} / \mathrm{L} \mathrm{NaCl}, 1 \mathrm{mmol} / \mathrm{L}$ dithiothreitol, $1 \mathrm{mmol} / \mathrm{L}$ sodium orthovanadate, $2 \mathrm{mmol} / \mathrm{L} \mathrm{MgCl} 2,1 \mathrm{mmol} / \mathrm{L}$ phenylmethylsulfonyl fluoride with $1 \%$ protease inhibitor cocktail, and $0.25 \%$ NP-40) on ice for 20 minutes followed by 15 passages through a 26-gauge syringe needle. The nuclei were pelleted by centrifugation at $12,000 \times g$ for 1 minute and resuspended in $0.4 \mathrm{~N} \mathrm{HCl}$ with $10 \%$ glycerol, rotated overnight at $4^{\circ} \mathrm{C}$. The acid-soluble fractions were collected by centrifugation at $12,000 \times g$ for 10 minutes, and precipitation with $20 \%$ trichloroacetic acid. The pellets were washed with acetone, dried, and dissolved in distilled water.

\section{Immunoblot Analysis}

Liver or cell nuclear proteins were extracted using 10\% Nonidet P-40 lysis buffer containing protease inhibitor cocktail (leupeptin, aprotinin, pepstatin, and phenylmethylsulfonyl fluoride). Protein concentrations were measured by a kit based on the Bradford method (Bio-Rad, Hercules, CA). Aliquots containing 15 to $60 \mu \mathrm{g}$ proteins were loaded onto a $8 \%$ to $12 \%$ SDS-polyacrylamide gel, transblotted onto a polyvinylidene difluoride membrane (Bio-Rad), blocked with 10\% nonfat dry milk in Tris-buffered saline with $0.1 \%$ Tween-20, and then incubated with rabbit or mouse polyclonal antibody against NF- $\kappa B$, HDACs (HDAC1, HDAC2, HDAC3, HDAC4), total histone, acetyl-histone H3 (Lys9) (AcH3K9, Cell Signaling), p300, or $\beta$-actin (Santa Cruz Biotechnologies, Santa Cruz, CA). The membrane then was incubated with horseradish peroxidase-conjugated donkey anti-rabbit or goat anti-mouse IgG (Cell Signaling). The protein bands were visualized with an enhanced chemiluminescence system (GE Health Care, Piscataway, NJ), and quantified by densitometry analysis.

\section{Immunofluorescence Microscopy}

H4IIEC3 cell chamber slides were fixed with cold methanol for 5 minutes at $-20^{\circ} \mathrm{C}$. The $\mathrm{H} 4$ IIEC3 cells then were incubated with rabbit polyclonal anti-NF- $\mathrm{B}$ or antiAcH3K9 antibody (Cell Signaling) overnight at $4^{\circ} \mathrm{C}$, followed by incubation with a Cy3-conjugated antibody (Jackson ImmunoResearch Laboratories, West Grove, PA) for 30 minutes at room temperature. The cell nuclei were counterstained by DAPI.

\section{Co-Immunoprecipitation}

The nuclear fraction of treated H4IIEC3 cells was isolated, and NF- $\mathrm{BB}$ and HDAC1, HDAC2 complex were immunoprecipitated. NF- $\kappa$ B antibody (1:1,000 dilution; Cell Signaling) was added to $400 \mu \mathrm{g}$ of protein in a final volume of $500 \mu \mathrm{L}$ and incubated at $4^{\circ} \mathrm{C}$ overnight. Protein A agarose beads (BD Biosciences, San Jose, CA) were added to each sample and rocked for 2 hours at $4^{\circ} \mathrm{C}$. The samples then were centrifuged at $13,000 \times g$ at $4^{\circ} \mathrm{C}$ for 5 minutes. The supernatant was discarded, and the beads were washed twice, pelleted by centrifugation, and resuspended by adding $20 \mu \mathrm{L}$ of $5 \times$ sample buffer, boiled, and resolved by SDS-PAGE as described earlier. To show the interaction of $\mathrm{NF}-\kappa \mathrm{B}$ protein with $\mathrm{HDAC} 1$ and HDAC2, immunoprecipitated NF- $\kappa$ B was blotted against HDAC1 or HDAC2 (Cell Signaling).

\section{ChIP Analysis}

H4IIEC3 cells were treated with $1 \mu \mathrm{mol} / \mathrm{L}$ TPEN with or without $50 \mu \mathrm{mol} / \mathrm{L}$ zinc for 3 days, and ChIP analysis was performed. Briefly, cell chromatins were prepared using an 

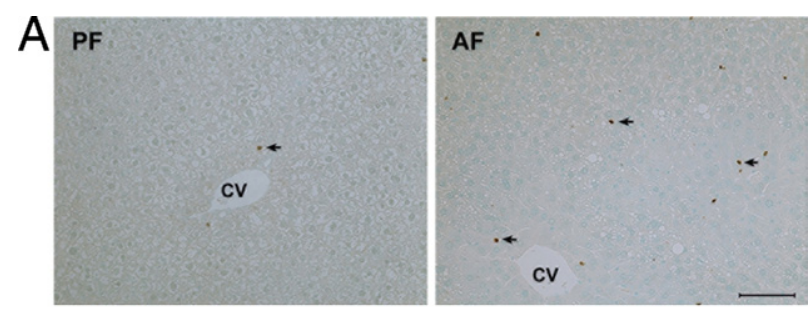

B

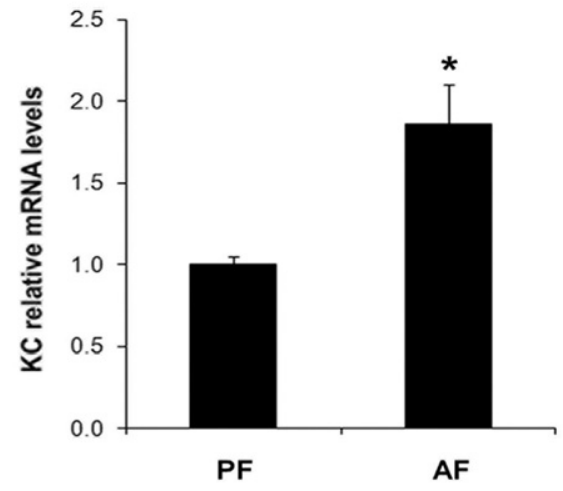

C

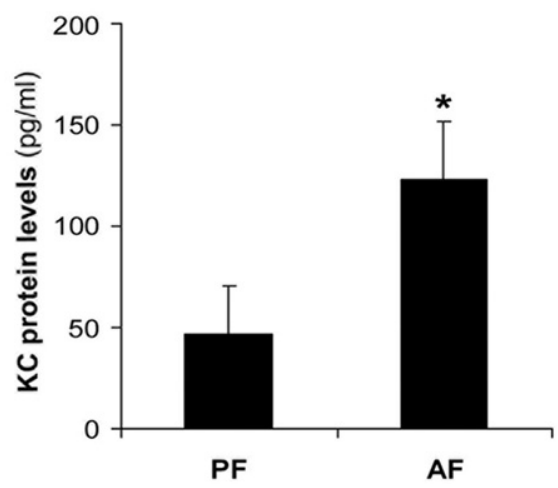

Figure 1. Hepatic neutrophil infiltration and IL- 8 analog expression in mice fed alcohol for 4 weeks. A: Neutrophils (arrows) in the liver detected by IHC. Scale bar $=50 \mu \mathrm{m}$. B: Real-time RT-PCR analysis of mRNA levels of IL-8 analog, mice KC. C: ELISA analysis of KC protein levels. Results in B and C are means $\pm \mathrm{SD}(n=6$ to 8$)$. Significant differences $\left({ }^{*} P<0.05\right)$ between pair-fed (PF) and alcohol-fed (AF) mice were determined by $t$-test. CV, central vein.

EZ-Zyme Chromatin Prep Kit (Upstate, Temecula, CA) according to the manufacturer's instructions. Immunoprecipitation was performed using $5 \mu \mathrm{g}$ of anti-AcH3K9, or anti$\mathrm{NF}-\kappa \mathrm{B}$ antibody (Cell Signaling). CINC-1 promoter-specific primers with the product including NF- $\kappa$ B-specific binding site were as follows: sense, 5' -GGGAATTTCCCTGGCCTGGAG-3' and antisense, 5'-GAAGCGAGCGGGTGGCTGAG-3'. Relative fold changes in histone 3 acetylation or $\mathrm{NF}-\kappa \mathrm{B}$ binding activity at CINC-1 promoter were determined by a semiquantitative assay using the $\mathrm{ABI} 7700$ detection system (Applied Biosystems, Foster City, CA) as recommended by the manufacturer.

\section{Real-Time RT-PCR}

The hepatic KC mRNA levels were assessed by real-time RT-PCR. In brief, the total RNA was isolated and reverse transcribed with the Moloney murine leukemia virus reverse transcriptase and oligo-dT primer. The forward (5'-
AACCGAAGTCATAGCCACAC-3') and reverse (5'-CAGACGGTGCCATCAGAG-3') primers were designed using Primer Express Software (Applied Biosystems). The SYBR green PCR Master Mix (Applied Biosystems) was used for real-time RT-PCR analysis. The relative differences of gene expression among groups were evaluated using cycle time values. The data were normalized to $\beta$-actin and expressed as relative changes, setting the values of control mice as one.

\section{Statistics}

All data are expressed as mean $\pm \mathrm{SD}(n=6$ to 8$)$. The data were analyzed by either analysis of variance and the Newman-Keuls multiple-comparison test for studies with more than 2 groups or the Student's $t$-test for studies with 2 groups. Differences between groups were considered significant at a $P$ value of $<0.05$.

\section{Results}

\section{Effects of Chronic Alcohol Feeding on Hepatic IL-8 Analog Expression, NF- $\kappa B$ Activation, and Histone 3 Acetylation}

As shown in Figure $1 \mathrm{~A}$, immunohistochemistry $(\mathrm{IHC})$ showed neutrophil infiltration in the livers of mice after chronic alcohol feeding for 4 weeks. In accordance, chronic alcohol feeding up-regulated IL-8 analog, the mouse KC, gene expression (Figure 1B), and increased its protein levels (Figure $1 C$ ) in the liver. Associations of $\mathrm{NF}-\kappa \mathrm{B}$ activation and histone acetylation with mouse $\mathrm{KC}$ expression were further determined. Chronic alcohol feeding activated $\mathrm{NF}-\kappa \mathrm{B}$, as indicated by increased nuclear translocation (Figure 2A). AcH3K9 was assessed by $\mathrm{IHC}$, and chronic alcohol feeding caused histone 3
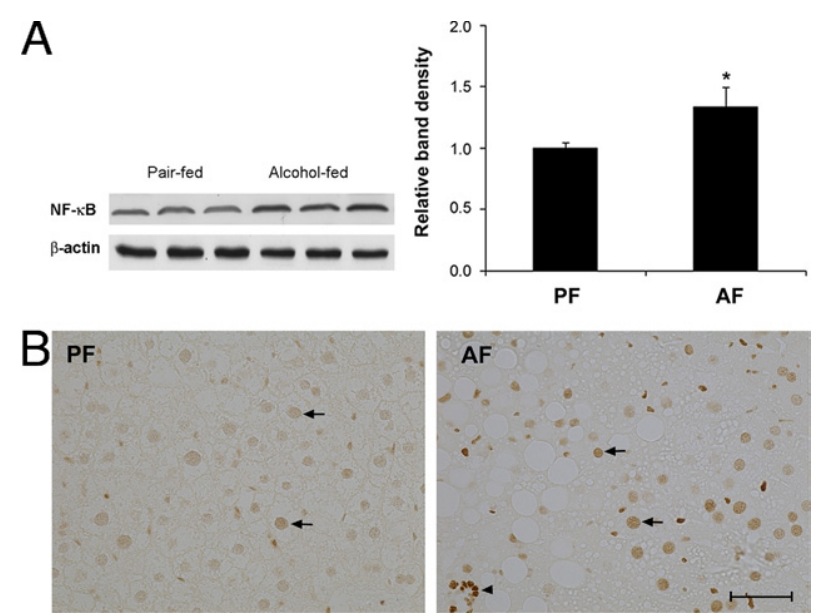

Figure 2. Hepatic NF- $\kappa \mathrm{B}$ activation and histone 3 acetylation in mice fed alcohol for 4 weeks. A: NF- $\kappa$ B activation detected by immunoblot of nuclear $\mathrm{NF}-\kappa \mathrm{B}$ protein. Significant differences $\left({ }^{*} P<0.05\right)$ in band density between pair-fed (PF) and alcohol-fed (AF) mice were determined by $t$-test $(n=3)$. B: Histone 3 acetylation at lysine 9 detected by IHC. Positive staining on hepatocytes and neutrophils are indicated by arrows and arrowheads, respectively. Scale bar $=100 \mu \mathrm{m}$. 
A

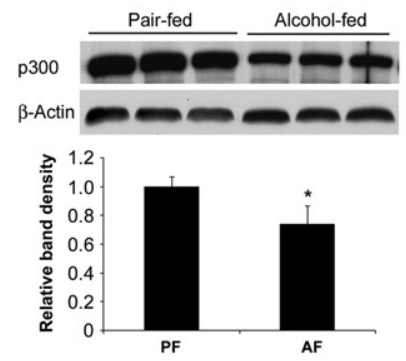

B

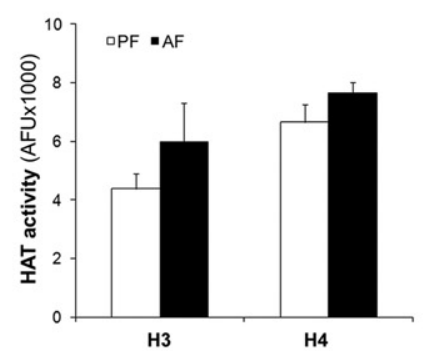

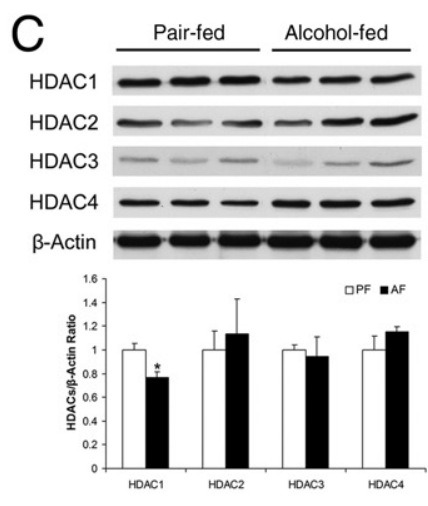

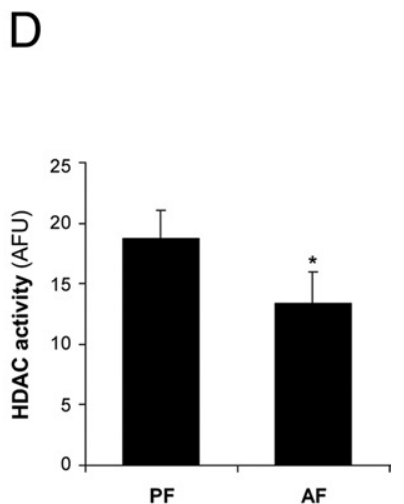

PF

Figure 3. Hepatic HAT and HDAC activities in mice fed alcohol for 4 weeks. A: Immunoblot of p300 protein. B: HAT activity was measured using a fluorimetric assay kit with acetyl-CoA and either a histone $\mathrm{H} 3$ or histone $\mathrm{H} 4$ substrate peptide. C: Immunoblot of HDAC proteins. D: HDAC activity was measured with a fluorimetric assay kit. Significant differences $\left({ }^{*} P<0.05\right)$ between pair-fed (PF) and alcohol-fed (AF) mice in A, C, and D were determined by $t$-test $(\mathbf{A}$ and $\mathbf{B}: n=$ 3; D: $n=6$ to 8). AFU, arbitrary fluoresence units.

hyperacetylation at lysine 9 in different cell types, including hepatocytes and neutrophils (Figure 2B).

\section{Effects of Chronic Alcohol Feeding on Hepatic HATs and HDACs}

Protein levels and activities of histone acetylation-related enzymes were determined. As shown in Figure 3A, chronic alcohol feeding reduced the protein levels of p300, a histone acetyltransferase, but did not significantly affect the hepatic total acetyltransferase activities to either histone $\mathrm{H} 3$ or $\mathrm{H} 4$ (Figure 3B). Chronic alcohol feeding reduced protein level of HDAC1, but did not affect HDAC2, HDAC3, or HDAC4 (Figure 3C). Total hepatic HDAC activities were decreased by chronic alcohol feeding (Figure 3D).

\section{Alcohol-Induced IL-8 Analog Production in Association with Accumulation of Acetaldehyde, ROS, and Free Zinc in Hepatoma Cells}

Alcohol exposure to VL-17A cells induced IL-8 production, which was normalized by either NAC or zinc (Figure 4A). Similarly, alcohol exposure also induced IL-8 analog, rat CINC-1, production in H4IIEC3 cells. Supplementation with NAC and/or zinc also normalized alcohol-induced CINC-1 production in H4IIEC3 cells. Because VL-17A cells produced a higher level of IL-8 in comparison with H4IIEC3 cells, acetaldehyde accumulation in the cell culture media was measured to define the role of alcohol metabolism in IL-8 production. In accordance with greater IL-8 production, acetaldehyde concentrations in the culture media were much higher in VL-17A cells ( $4383 \mathrm{ng} / 10^{6}$ cells) than H4lIEC3 cells ( $975 \mathrm{ng} / 10^{6}$ cells).

To determine whether zinc release is associated with alcohol-induced chemokine production, cellular ROS and free zinc were measured in H4IIEC3 cells. As shown in Figure 4C, alcohol exposure caused accumulation of ROS, which was normalized by NAC, and partially attenuated by zinc. Alcohol exposure also increased cellular free zinc levels as indicated by a fluorescence zinc probe, FluoZin-3 (Figure 4D). Both NAC and zinc supplementation attenuated the alcohol-increased cellular free zinc level.

\section{Zinc Deprivation Induces IL-8 Analog Production in Hepatoma Cells}

To define the role of zinc deprivation in IL-8 analog production, H4IIEC 3 cells were treated with TPEN for 3 days. Zinc deprivation by TPEN caused CINC-1 production,
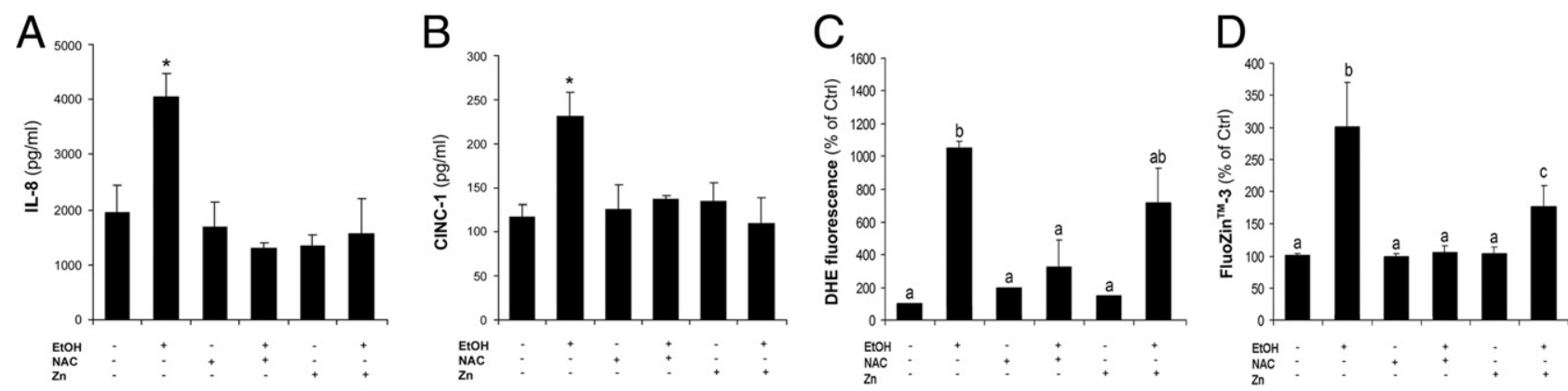

Figure 4. Alcohol-induced IL-8 analog production in hepatoma cells. A: ELISA analysis of IL-8 production from VL-17A cells. B: ELISA analysis of IL-8 analog, rat CINC-1, production in H4IIEC3 cells. C: Cellular ROS accumulation in H4IIEC3 cells as measured by DHE fluorescence probe. D: Free zinc levels in H4IIEC3 cells detected by FluoZin-3, a free zinc probe. Results are means \pm SD ( $n=4$ to 6 ). Significant differences ( $\left.{ }^{*} P<0.05\right)$ among a, b, and c were determined by analysis of variance and indicated by asterisk in $\mathbf{A}$ and $\mathbf{B}$ and letters in $\mathbf{C}$ and $\mathbf{D}$ 

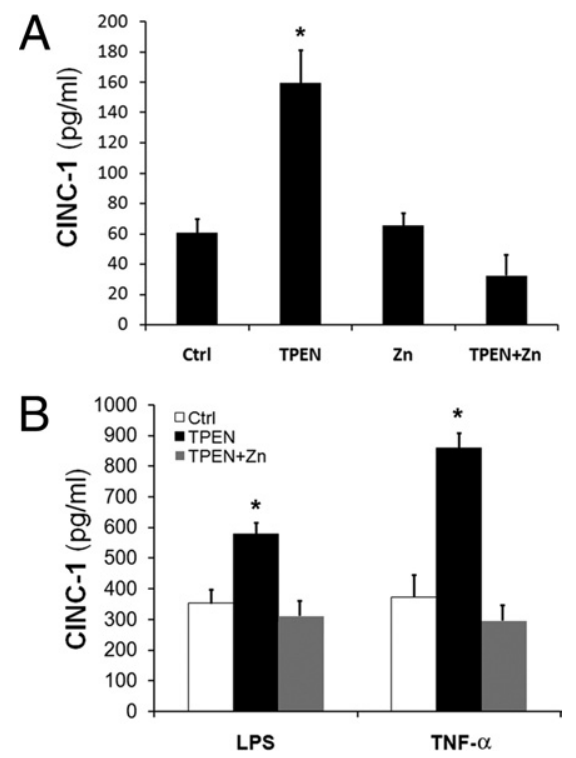

Figure 5. Zinc deprivation-induced IL-8 analog production in H4IIEC3 cells A: TPEN-induced CINC-1 production (ELISA). B: TPEN-induced priming effect on LPS or TNF- $\alpha$ induction of IL-8 analog, rat CINC-1, production (ELISA). Significant differences $\left({ }^{*} P<0.05\right)$ are determined by analysis of variance. Ctrl, control; LPS, lipopolysaccharide.

which was normalized by adding zinc back to the medium (Figure 5A). To determine whether zinc deprivation has a priming effect on CINC-1 production, H4IIEC3 cells first were treated with TPEN for 2 days, followed by LPS or TNF- $\alpha$ exposure for 24 hours. As shown in Figure 5B, zinc deprivation showed an additive effect on LPS-induced CINC-1 production, and a synergistic effect on TNF- $\alpha-$ induced CINC-1 production.

\section{Effects of Zinc Deprivation on HDACs and Histone Acetylation in Hepatoma Cells}

Zinc deprivation by TPEN dramatically decreased HDAC activity, which was prevented by adding zinc back to the medium (Figure 6A). Immunoblot analysis showed that TPEN treatment increased protein levels of HDAC1, HDAC3, and HDAC4, but did not affect HDAC2 (Figure $6 B)$. Zinc supplementation normalized the effects of TPEN on HDAC protein levels (Figure 6B). Histone 3 acetylation at lysine 9 was assessed by immunoblot analysis and immunocytochemistry. As shown in Figure 7A, TPEN treatment significantly increased histone 3 acetylation at lysine 9, without affecting the total protein level of histone 3. TPEN-induced histone 3 hyperacetylation was further confirmed by immunocytochemical staining, as indicated by intensified nuclear staining (Figure 7B).

\section{Effects of Zinc Deprivation on NF- $\kappa$ B Activation and $N F-\kappa B-H D A C$ Interaction in Hepatoma Cells}

As shown by immunocytochemistry, NF- $\kappa \mathrm{B}$ proteins mainly are located in the cytoplasm in H4IIEC3 cells under normal conditions (Figure 8A). Zinc deprivation by
TPEN induced NF- $\kappa \mathrm{B}$ activation as indicated by nuclear translocation, reduced cytoplasmic staining, and increased nuclear staining. Interactions between NF- $\kappa \mathrm{B}$ and HDACs were assessed by co-immunoprecipitation. Zinc deprivation by TPEN increased nuclear NF- $\kappa \mathrm{B}$, although it decreased HDAC1 and HDAC2 binding to NF- $\kappa \mathrm{B}$ (Figure 8B). Zinc supplementation prevented the TPEN effects on NF- $\kappa \mathrm{B}$ and HDAC binding to NF- $\kappa \mathrm{B}$.

\section{Effects of Zinc Deprivation on NF- $\kappa$ B Binding and Histone Acetylation at CINC-1 Promoter Region in Hepatoma Cells}

A ChIP assay was performed to determine whether zinc deprivation affects $\mathrm{NF}-\kappa \mathrm{B}$ binding to the CINC-1 promoter and modulates histone acetylation at the same time. A ChIP assay with $\mathrm{AcH} 3 \mathrm{~K} 9$ immunoprecipitation showed that TPEN increased histone 3 acetylation at lysine 9 at the CINC-1 promoter region in H4IIEC3 cells (Figure 9A). Similarly, a ChIP assay with NF- $\kappa$ B immunoprecipitation showed that zinc deprivation by TPEN dra-
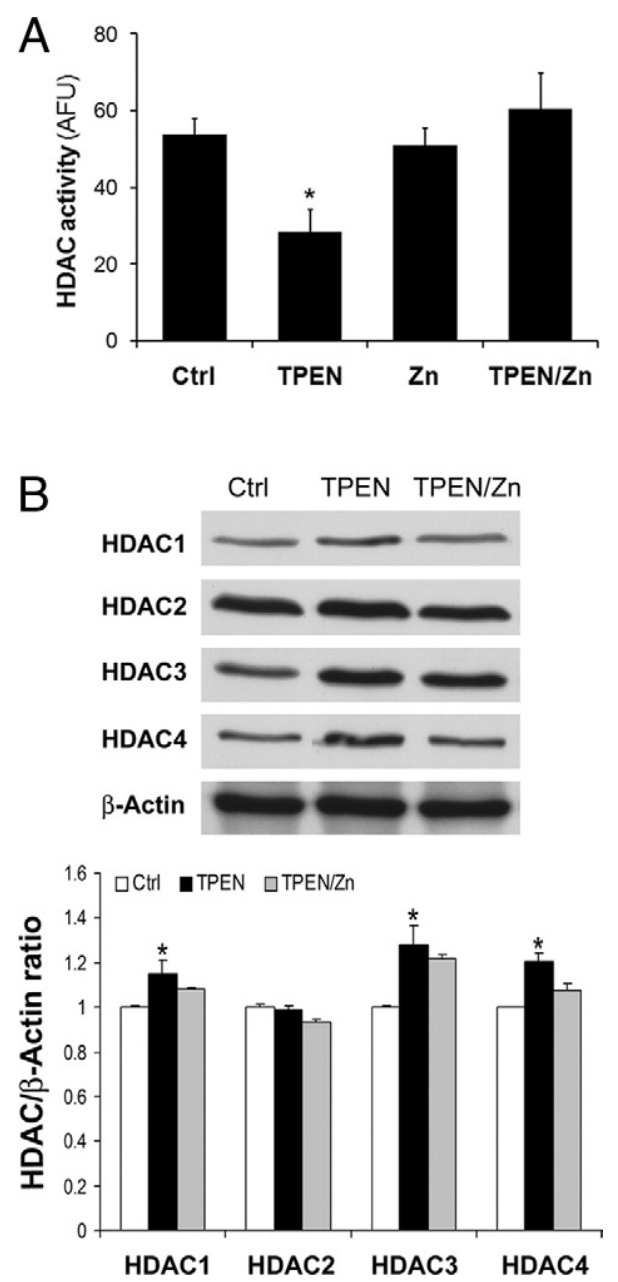

Figure 6. Inactivation of HDAC by zinc deprivation in H4IIEC3 cells. A: HDAC activity was measured with a fluorimetric assay kit. B: Immunoblot of HDAC proteins. Significant increases $\left({ }^{*} P<0.05\right)$ by TPEN were determined by analysis of variance. AFU, arbitrary fluoresence units; Ctrl, control. 
A

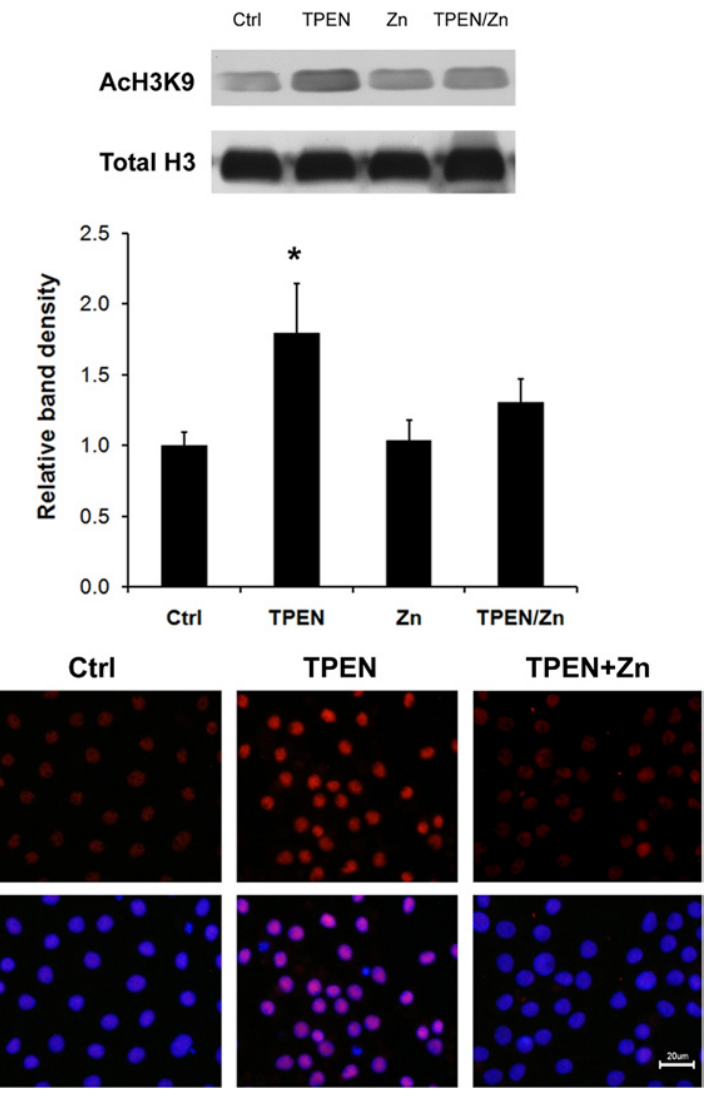

Figure 7. Histone 3 hyperacetylation by zinc deprivation in H4IIEC3 cells. A: Immunoblot of AcH3K9. B: Immunofluorescence microscopy of AcH3K9. Red: NF- $\kappa$ B; blue: DAPI counterstaining of nuclei. Significant increases $\left({ }^{*} P<\right.$ $0.05)$ by TPEN were determined by analysis of variance $(n=3)$. Ctrl, control.

matically increased NF- $\kappa \mathrm{B}$ binding to the CINC-1 promoter (Figure 9B). Zinc supplementation prevented TPEN-induced NF- $\kappa$ B binding.

\section{Discussion}

Results obtained in the present study showed that histone 3 acetylation at lysine 9 caused by HDAC inactivation was associated with $\mathrm{NF}-\kappa \mathrm{B}$ activation and mouse IL-8 expression in the liver after chronic alcohol exposure. Studies with VL-17A cells (ADH/CYP2E1-overexpressing HepG2 cells) and H4IIEC3 cells showed that alcohol-induced IL-8 expression in hepatocytes was dependent on oxidative stress and zinc deprivation. Experimental zinc deprivation not only induced IL-8 expression, but also primed LPS- and TNF- $\alpha$-induced IL- 8 expression in H4IIEC3 cells. The inhibitory effect of zinc deprivation on HDAC activity, rather than on protein levels, indicates a pivotal role for zinc in maintaining HDAC enzyme activity. Zinc deprivation caused NF- $\kappa$ B nuclear translocation, and reduced HDAC1/HDAC2 binding to $\mathrm{NF}-\kappa \mathrm{B}$. Ultimately, zinc deprivation increased histone 3 hyperacetylation at the IL-8 promoter as well as NF- $\kappa \mathrm{B}$ binding to IL-8 promoter. These data suggest that inactivation of HDAC owing to zinc deprivation is a novel mechanism of up-regulating IL-8 gene expression in alcoholic liver disease.

Chronic alcohol exposure to rodents has been shown to increase hepatic IL-8 analog (mouse KC or rat CINC-1) levels in association with neutrophil infiltration, ${ }^{1,9,10}$ and the hepatocyte is one of the cell types responsible for IL-8 production. ${ }^{37}$ Mechanistic studies have indicated that alcohol metabolism alone is able to trigger IL-8 production in hepatocytes, although results from different reports are controversial. Alcohol exposure to human and rat hepatocytes has been shown to induce chemotactic activity for neutrophils, which was attenuated by inhibition of alcohol metabolism with ADH inhibitor. ${ }^{11,12}$ These results suggest that alcohol-induced IL-8 production in hepatocytes is dependent on acetaldehyde and/or oxidative stress. HepG2 cell culture showed that acetaldehyde and LPS, but not alcohol, induced oxidative stress and IL-8 production. ${ }^{15}$ Another cell culture study with primary rat hepatocytes showed that rat IL-8 analog expression was stimulated by cytokines, in particular TNF- $\alpha$, but not by LPS or alcohol. ${ }^{38}$ Although there were no evidence to show that whether or not the primary hepatocytes maintained their alcohol metabolic capacity, HepG2 cells are known to lack ADH and CYP2E1. ${ }^{36}$ Thus, HepG2 cells may not generate a significant amount of acetaldehyde and ROS, which could explain why alcohol exposure failed to induce oxidative stress and IL-8 production in HepG2 cells. The hepatoma cell lines of

A
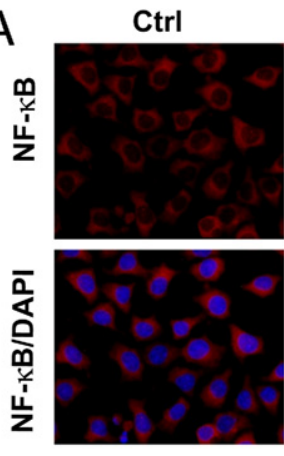

B
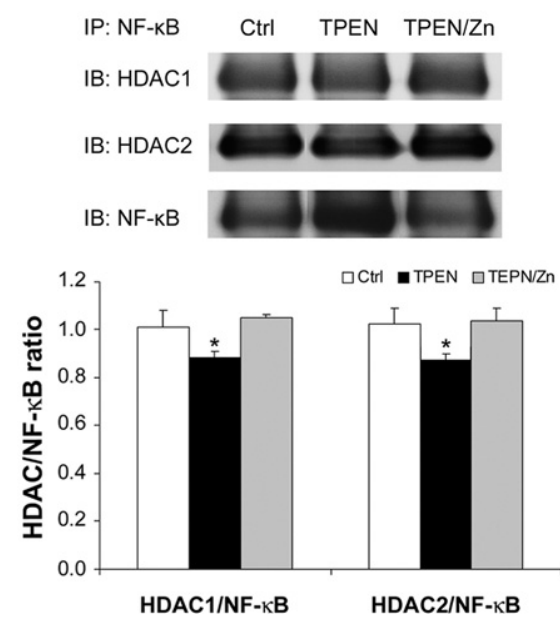

Figure 8. Zinc deprivation-induced NF- $\kappa$ B activation in H4IIEC3 cells. A Immunocytochemistry of nuclear translocation of NF- $\kappa$ B after TPEN treatment at $1 \mu \mathrm{mol} / \mathrm{L}$ for 3 days. Red: NF- $\kappa$ B; blue: DAPI counterstaining of nuclei. B: Immunoprecipitation (IP) assay followed by immunoblotting (IB) of NF- $\kappa \mathrm{B}$ binding with HDAC1 and HDAC2. Significant decreases $\left({ }^{*} P<0.05\right)$ by TPEN were determined by analysis of variance $(n=3)$. Ctrl, control 
A
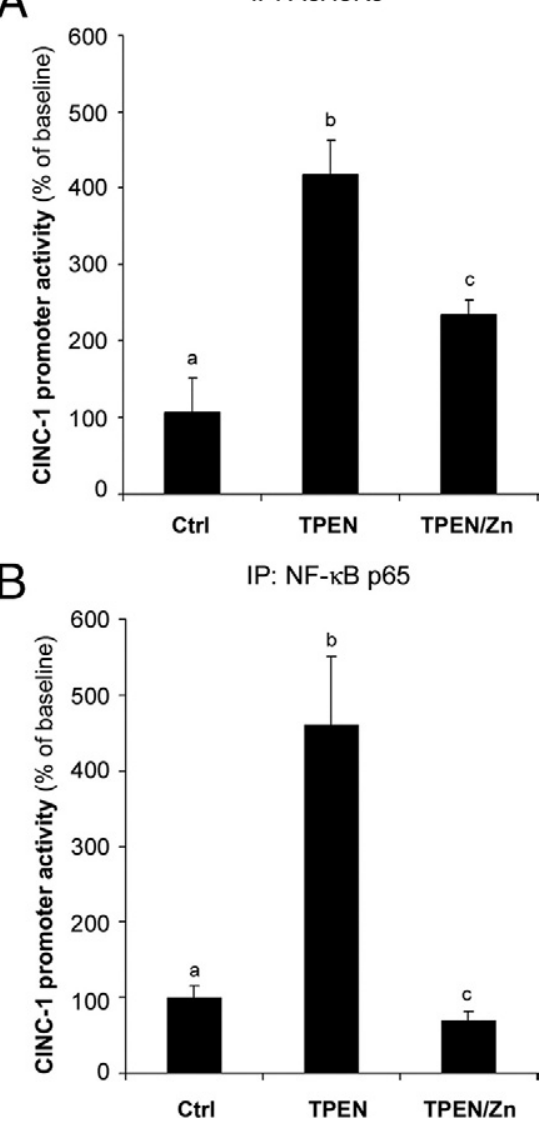

Figure 9. Effects of zinc deprivation on histone 3 acetylation and NF- $\kappa \mathrm{B}$ binding at CINC-1 promoter in H4IIEC3 cells. A: Chromatin immunoprecipitation (ChIP) analysis of histone 3 acetylation at CINC-1 promoter. IP with anti-AcH3K9 antibody and real-time RT-PCR assay of CINC-1 expression. B: ChIP analysis of $\mathrm{NF}-\kappa \mathrm{B}$ binding to $\mathrm{CINC}-1$ promoter. Immunoprecipitation (IP) with anti-NF- $\kappa \mathrm{B}$ p65 antibody and real-time RT-PCR assay of CINC-1 expression. Significant increases $\left({ }^{*} P<0.05\right)$ among $\mathrm{a}, \mathrm{b}$, and $\mathrm{c}$ by TPEN were determined analysis of variance $(n=4)$. Ctrl, control.

VL-17A and H4IIEC3 used in the present study are known to express alcohol-metabolizing enzymes. ${ }^{36,39}$ Alcohol metabolism generates acetaldehyde, which has been shown to contribute to ROS generation significantly. ${ }^{40}$ The present study showed that VL-17A cells produced a greater amount of acetaldehyde in association with IL-8 analog production in comparison with H4IIEC3 cells as a result of overexpression of $\mathrm{ADH}$ and CYP2E1. These results suggest that alcohol exposure may stimulate IL-8 production in hepatocytes, at least partially, through generation of acetaldehyde and oxidative stress.

Oxidative stress has been suggested as an important mechanism underlying LPS-induced cytokine production in Kupffer cells. ${ }^{41}$ Activation of NF- $\kappa \mathrm{B}$ is an important mechanism that links oxidative stress with cytokine gene expression. Hepatocytes are the major source of acetaldehyde and ROS generation after alcohol exposure, but the link between oxidative stress and IL-8 production in hepatocytes is poorly understood. Treatments with acetaldehyde and LPS induced IL-8 production in HepG2 cells; however, only acetaldehyde significantly increased lipid peroxidation. ${ }^{14}$ Alcohol exposure to VL-17A cells and H4IIEC3 cells in the present study induced IL-8 an- alog production in association with oxidative stress and zinc release. Because supplementation with NAC or zinc prevented alcohol-induced IL-8 production, oxidative stress and zinc release are like key mediators. Although $\mathrm{ROS}$ are known to induce IL-8 production via activation of $\mathrm{NF}-\kappa \mathrm{B}$, induction of IL-8 analog in H4IIEC3 cells by zinc deprivation suggests that zinc release could be a downstream event of ROS in IL-8 gene expression. Previous reports have shown that both $\mathrm{ROS}$ and acetaldehyde can release zinc from zinc proteins. ${ }^{40,42,43}$ The present study showed that zinc deprivation inhibited HDAC activity without affecting protein levels, suggesting zinc release from the zinc figure. However, chronic alcohol exposure inhibited HDAC activity in association with a reduction of the HDAC1 protein level, suggesting that alcohol may inhibit HDAC activity at both the transcriptional level (protein expression) and the posttranscriptional level (zinc release).

Although deacetylation of histones is the major mechanism of transcriptional regulation by HDACs, recent studies have shown that deacetylation of $\mathrm{NF}-\kappa \mathrm{B}$ by HDACs affect NF- $\kappa$ B activity. The present study showed that zinc deprivation not only affected HDAC activity and $\mathrm{NF}-\kappa \mathrm{B}$ nuclear translocation, but also interfered with HDAC-NF- $\kappa$ B interaction. Because deacetylation of $\mathrm{NF}-\kappa \mathrm{B}$ by HDACs inhibits NF- $\kappa \mathrm{B}$ activity, reduction of HDAC binding to NF- $\kappa$ B by zinc deprivation may impact $\mathrm{NF}-\kappa \mathrm{B}$ activity. These results suggest that HDAC inactivation as a result of zinc release may facilitate NF- $\kappa$ Bmediated transcription by directly affecting acetylation status of both histones and NF- $\kappa \mathrm{B}$.

Zinc deprivation not only activated $\mathrm{NF}-\kappa \mathrm{B}$, but also inhibited HDAC activity in H4IIEC3 cells. Because HDACs play a critical role in histone acetylation and gene transcription, an epigenetic mechanism likely is involved in zinc regulation of $\mathrm{IL}-8$ expression.

Increasing evidence suggests that inhibition of HDACs under oxidative stress conditions is a key epigenetic mechanism, even in IL-8 gene expression in lung inflammatory disease. ${ }^{16-18}$ Oxidants $\left(\mathrm{H}_{2} \mathrm{O}_{2}\right)$ or cytokines (TNF- $\alpha$ and IL-1 $\beta$ ) have been shown to suppress HDAC activity associated with IL-8 production in alveolar epithelial cells. ${ }^{23-25}$ Pharmaceutical inhibition of HDAC by trichostatin A not only stimulated IL-8 production via in-

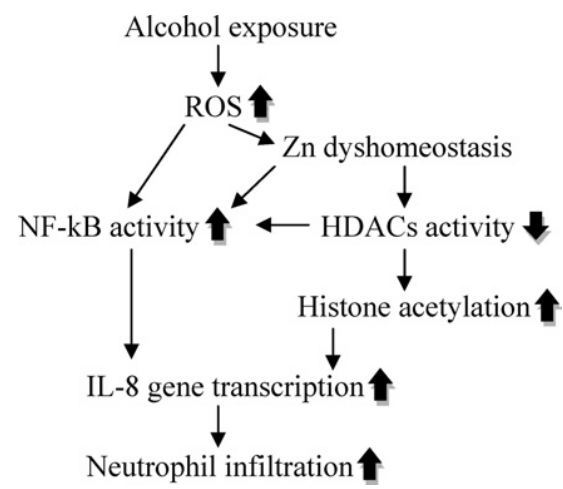

Figure 10. Working hypothesis on zinc deprivation and subsequent modulation of epigenetic control of IL-8 analog expression in alcoholic hepatitis. 
duction of histone acetylation, but also had a synergistic effect on oxidant- or TNF- $\alpha$-induced IL-8 expression. ${ }^{23-25}$ Because trichostatin $A$ did not significantly affect NF- $\kappa$ B activation, these studies suggest a causal role of HDACs in regulating IL-8 production. On the other hand, preservation of HDAC activity by glutathione compounds including NAC and glutathione monoethyl ester reversed oxidant- or cigarette smoke-induced IL-8 expression in alveolar epithelial cells and macrophages. ${ }^{40,42}$ A clinical study showed that induction of HDAC activity is involved in the anti-inflammatory action of theophylline. ${ }^{43}$ Although histone acetylation has been shown repeatedly in alcohol-induced liver injury, ${ }^{26-30}$ the mechanistic link between HDACs and IL-8 expression has not been defined. The present study showed a reduction of HDAC activity, rather than increased HAT activity, was associated with chronic alcohol exposure-induced histone 3 hyperacetylation at lysine 9 in multiple cell populations including hepatocytes. Interestingly, alcohol only reduced the protein level of HDAC1, but not HDAC2, HDAC3, or HDAC4. Because our previous reports have shown that chronic alcohol exposure causes zinc deficiency in the liver, reduction of HDAC activity after alcohol exposure is most likely owing to reduced zinc coordination to HDAC proteins. Indeed, experimental zinc deprivation showed a significant inhibition of HDAC activity even with increased protein levels of HDACs, indicating a key role of zinc in maintenance of HDAC activity. HDAC inactivation owing to zinc deprivation not only caused histone 3 acetylation at lysine 9 at the CINC-1 promoter region, but also limited their binding to $N F-\kappa B$, an inhibitory mechanism of NF- $\kappa$ B activation. These findings for the first time show that zinc deprivation up-regulates IL-8 analog expression via inactivation of HDACs.

In summary, the present study showed that alcohol exposure induces hepatocyte IL-8 expression in an epigenetic manner and zinc deprivation mediates alcohol action via inactivation of HDACs (Figure 10). Chronic alcohol exposure caused histone 3 acetylation at lysine 9 in association with IL-8 analog production in the liver, and inactivation of HDACs was responsible for histone 3 hyperacetylation. Cell culture studies showed that alcohol exposure induced IL-8 analog production in hepatocytes via an oxidative stress mechanism and zinc release is a downstream molecular event of oxidative stress. Inactivation of HDACs owing to zinc deprivation led to histone 3 acetylation at lysine 9, thereby facilitating NF- $\kappa$ B-mediated IL-8 analog transcription. These results suggest that inactivation of HDACs as a result of zinc deprivation is a novel mechanism underlying alcohol-induced IL-8 analog expression in hepatocytes.

\section{Acknowledgments}

We thank Xinguo Sun for technical assistance and Marion McClain for review of this manuscript.

\section{References}

1. Bautista AP: Neutrophilic infiltration in alcoholic hepatitis. Alcohol 2002, 27:17-21

2. Ramaiah SK, Jaeschke $\mathrm{H}$ : Hepatic neutrophil infiltration in the pathogenesis of alcohol-induced liver injury. Toxicol Mech Methods 2007. 17:431-440

3. Jaeschke $\mathrm{H}$ : Neutrophil-mediated tissue injury in alcoholic hepatitis Alcohol 2002, 27:23-27

4. Bird G: Interleukin-8 in alcoholic liver disease. Acta Gastroenterol Belg 1994, 57:255-259

5. McClain CJ, Song Z, Barve SS, Hill DB, Deaciuc I: Recent advances in alcoholic liver disease. IV. Dysregulated cytokine metabolism in alcoholic liver disease. Am J Physiol 2004, 287:G497-G502

6. Huang YS, Chan CY, Wu JC, Pai CH, Chao Y, Lee SD: Serum levels of interleukin-8 in alcoholic liver disease: relationship with disease stage, biochemical parameters and survival. J Hepatol 1996, 24: 377-384

7. Dominguez M, Miquel R, Colmenero J, Moreno M, García-Pagán JC, Bosch J, Arroyo V, Ginès P, Caballería J, Bataller R: Hepatic expression of CXC chemokines predicts portal hypertension and survival in patients with alcoholic hepatitis. Gastroenterology 2009, 136: 1639-1650

8. Afford SC, Fisher NC, Neil DA, Fear J, Brun P, Hubscher SG, Adams $\mathrm{DH}$ : Distinct patterns of chemokine expression are associated with leukocyte recruitment in alcoholic hepatitis and alcoholic cirrhosis. J Pathol 1998, 186:82-89

9. Zhou Z, Liu J, Song Z, McClain CJ, Kang YJ: Zinc supplementation inhibits hepatic apoptosis in mice subjected to a long-term ethanol exposure. Exp Biol Med (Maywood) 2008, 233:540-548

10. Ohki E, Kato S, Ohgo H, Mizukami T, Fukuda M, Tamai $H$, Okamura Y, Matsumoto M, Suzuki H, Yokoyama H, Ishii H: Effect of chronic ethanol feeding on endotoxin-induced hepatic injury: role of adhesion molecules on leukocytes and hepatic sinusoid. Alcohol Clin Exp Res 1998, 22:129S-132S.

11. Shiratori $Y$, Takada H, Hikiba $Y$, Okano K, Niwa $Y$, Matsumura M, Komatsu Y, Omata M: Increased release of KC/gro protein, intercrine cytokine family, from hepatocytes of the chronically ethanol fed rats. Biochem Biophys Res Commun 1993, 197:319-325

12. Shiratori $Y$, Hikiba $Y$, Mawet E, Niwa $Y$, Matsumura M, Kato N, Shiina $\mathrm{S}$, Tada M, Komatsu $\mathrm{Y}$, Kawabe $\mathrm{T}$ : Modulation of $\mathrm{KC} /$ gro protein (interleukin-8 related protein in rodents) release from hepatocytes by biologically active mediators. Biochem Biophys Res Commun 1994, 203:1398-1403

13. Shiratori $Y$, Takada $H$, Hai K, Kiriyama H, Mawet E, Komatsu Y, Niwa $Y$, Matsumura M, Shiina S, Kawase T: Effect of anti-allergic agents on chemotaxis of neutrophils by stimulation of chemotactic factor released from hepatocytes exposed to ethanol. Dig Dis Sci 1994, 39:1569-1575

14. Gómez-Quiroz L, Bucio L, Souza V, Escobar C, Farfán B, Hernández E, Konigsberg M, Vargas-Vorackova F, Kershenobich D: Interleukin 8 response and oxidative stress in HepG2 cells treated with ethanol, acetaldehyde or lipopolysaccharide. Hepatol Res 2003, 26:134-141

15. Joshi-Barve S, Barve SS, Butt W, Klein J, McClain CJ: Inhibition of proteasome function leads to NF-kappaB-independent IL-8 expression in human hepatocytes. Hepatology 2003, 38:1178-1187

16. Joshi-Barve S, Barve SS, Amancherla K, Gobejishvili L, Hill D, Cave M, Hote P, McClain CJ: Palmitic acid induces production of proinflammatory cytokine interleukin-8 from hepatocytes. Hepatology 2007, 46:823-830

17. Chen LF, Fischle W, Verdin E, Greene WC: Duration of nuclear NF-kappaB action regulated by reversible acetylation. Science 2001, 293:1653-1657

18. Adcock IM, Ito K, Barnes PJ: Histone deacetylation: an important mechanism in inflammatory lung diseases. COPD 2005, 2:445-455

19. Ito K, Charron CE, Adcock IM: Impact of protein acetylation in inflammatory lung diseases. Pharmacol Ther 2007, 116:249-265

20. Yang XJ, Seto E: HATs and HDACs: from structure, function and regulation to novel strategies for therapy and prevention. Oncogene 2007, 26:5310-5318

21. Rajendrasozhan S, Yang SR, Edirisinghe I, Yao H, Adenuga D, Rahman I: Deacetylases and NF-kappaB in redox regulation of cigarette 
smoke-induced lung inflammation: epigenetics in pathogenesis of COPD. Antioxid Redox Signal 2008, 10:799-811

22. Rahman I: Oxidative stress, transcription factors and chromatin remodeling in lung inflammation. Biochem Pharmacol 2002, 64: 935-942

23. Rahman I, Marwick J, Kirkham P: Redox modulation of chromatin remodeling: impact on histone acetylation and deacetylation. NFkappaB and pro-inflammatory gene expression. Biochem Pharmacol 2004, 68:1255-1267

24. Rahman I, Gilmour PS, Jimenez LA, MacNee W: Oxidative stress and TNF-alpha induce histone acetylation and NF-kappaB/AP-1 activation in alveolar epithelial cells: potential mechanism in gene transcription in lung inflammation. Mol Cell Biochem 2002, 234-235:239-248

25. Tomita K, Barnes PJ, Adcock IM: The effect of oxidative stress on histone acetylation and IL-8 release. Biochem Biophys Res Commun 2003, 301:572-577

26. Ito K, Hanazawa T, Tomita K, Barnes PJ, Adcock IM: Oxidative stress reduces histone deacetylase 2 activity and enhances IL-8 gene expression: role of tyrosine nitration. Biochem Biophys Res Commun 2004, 315:240-245

27. Shukla SD, Velazquez J, French SW, Lu SC, Ticku MK, Zakhari S: Emerging role of epigenetics in the actions of alcohol. Alcohol Clin Exp Res 2008, 32:1525-1534

28. Park PH, Miller R, Shukla SD: Acetylation of histone H3 at lysine 9 by ethanol in rat hepatocytes. Biochem Biophys Res Commun 2003, 306:501-504

29. Park PH, Lim RW, Shukla SD: Involvement of histone acetyltransferase (HAT) in ethanol-induced acetylation of histone $\mathrm{H} 3$ in hepatocytes: potential mechanism for gene expression. Am J Physiol Gastrointest Liver Physiol 2005, 289:G1124-G1136

30. Kim JS, Shukla SD: Acute in vivo effect of ethanol (binge drinking) on histone $\mathrm{H} 3$ modifications in rat tissues. Alcohol Alcohol 2006, 41:126-132

31. Choudhury M, Shukla SD: Surrogate alcohols and their metabolites modify histone $\mathrm{H} 3$ acetylation: involvement of histone acetyl transferase and histone deacetylase. Alcohol Clin Exp Res 2008, 32: $829-839$
32. Kiilerich S, Dietrichson O, Loud FB, Naestoft J, Christoffersen P, Juhl E, Kjems G, Christiansen C: Zinc depletion in alcoholic liver diseases. Scand J Gastroenterol 1980, 15:363-367

33. McClain CJ, Antonow DR, Cohen DA, Shedlofsky S: Zinc metabolism in alcoholic liver disease. Alcohol Clin Exp Res 1986, 10:582-589

34. Zhou Z, Wang L, Song Z, Saari JT, McClain CJ, Kang YJ: Zinc supplementation prevents alcoholic liver injury in mice through attenuation of oxidative stress. Am J Pathol 2005, 166:1681-1690

35. Zhong W, McClain CJ, Cave M, Kang YJ, Zhou Z: The role of zinc deficiency in alcohol-induced intestinal barrier dysfunction. Am J Physiol Gastrointest Liver Physiol 2010, 298:G625-G633

36. Donohue TM, Osna NA, Clemens DL: Recombinant Hep G2 cells that express alcohol dehydrogenase and cytochrome P450 2E1 as a model of ethanol-elicited cytotoxicity. Int J Biochem Cell Biol 2006, 38:92-101

37. Roll FJ, Bissell DM, Perez HD: Human hepatocytes metabolizing ethanol generate a non-polar chemotactic factor for human neutrophils. Biochem Biophys Res Commun 1986, 137:688-694

38. Ohkubo K, Masumoto T, Horiike N, Onji M: Induction of CINC (interleukin-8) production in rat liver by non-parenchymal cells. J Gastroenterol Hepatol 1998, 13:696-702

39. Crabb DW, Stewart MJ, Xiao Q: Hormonal and chemical influences on the expression of class 2 aldehyde dehydrogenases in rat $\mathrm{H} 4$ IIEC3 and human HuH7 hepatoma cells. Alcohol Clin Exp Res 1995, 19: 1414-1419

40. Li SY, Li Q, Shen JJ, Dong F, Sigmon VK, Liu Y, Ren J: Attenuation of acetaldehyde-induced cell injury by overexpression of aldehyde dehydrogenase-2 (ALDH2) transgene in human cardiac myocytes: role of MAP kinase signaling. J Mol Cell Cardiol 2006, 40:283-294.

41. Bautista AP: Impact of alcohol on the ability of Kupffer cells to produce chemokines and its role in alcoholic liver disease. J Gastroenterol Hepatol 2000, 15:349-356

42. Hao $Q$, Maret W: Aldehydes release zinc from proteins. A pathway from oxidative stress/lipid peroxidation to cellular functions of zinc. FEBS J 2006, 273:4300-4310.

43. Webster KA, Prentice H, Bishopric NH: Oxidation of zinc finger transcription factors: physiological consequences. Antioxid Redox Signal 2001, 3:535-548 